\title{
TECNOLOGIAS DIGITAIS DE COMUNICAÇÃO E O ENSINO E APRENDIZAGEM NO ENSINO SUPERIOR
}

Katia Denise Costa Berni, Universidade Federal de Pelotas, katiaberni13@gmail.com Christiano Martino Otero Avila, Universidade Federal de Pelotas, christiano.avila@ufpel.edu.br Rosaria Ilgenfritz Sperotto, Universidade Federal de Pelotas, ris1205@gmail.com Xavier Muianga, Universidade Eduardo Mondlane, xmuianga@gmail.com

\begin{abstract}
Resumo
Este artigo apresenta os esforços do grupo de pesquisa Comunicação, Cultura, Tecnologias e Modos de Subjetivação (CocTec) $)^{1}$, da Faculdade de Educação da Universidade Federal de Pelotas (UFPel) em mapear o uso das mídias digitais por seus alunos, nativos digitais, nascidos após 1990, problematizando o uso deste dispositivo como apoio ao ensino e aprendizagem e construção de conhecimento no ensino superior. São sujeitos desta pesquisa os alunos da UFPel nos cursos de bacharelado e licenciatura matriculados no $2^{\mathrm{a}}$ semestre de 2013 (exceto os ingressantes). Os dados foram capturados por um instrumento online disponibilizado junto à matrícula do aluno. Com esta pesquisa constatou-se que tanto para estudantes como para os professores os Sites de Redes Sociais (SRS) apresenta-se como uma ferramenta tecnológica pedagógica, servindo para: pesquisar conteúdos; realizar tarefas escolares; comunicar-se, enviar e receber materiais através do serviço de e-mail. Por outro lado, os SRS avançam e alargam as possibilidades de comunicação e interação. O presente texto está dividido em uma parte introdutória sobre o aluno da contemporaneidade, um debate sobre a utilização dos SRS no ensino e aprendizagem, um relato das principais revelações da pesquisa e considerações finais. Palavras-chaves: Ensino e Aprendizagem; Sites de Rede Sociais; Educação; Nativos digitais.
\end{abstract}

\section{DIGITAL COMMUNICATION TECHNOLOGIES AND TEACHING IN HIGHER EDUCATION}

\begin{abstract}
This article presents the efforts of the research group Communication, Culture, Technology and Modes of Subjectivity ( CocTec ), Faculty of Education, Federal University of Pelotas ( UFPel ) to map the use of digital media by his students, digital natives, born after 1990 questioning the use of such device to support teaching and learning and knowledge construction in higher education. Are subjects of research students UFPel in bachelor's degree and enrolled in the 2nd half of 2013 ( excluding freshmen). Data were captured by an online tool provided by the enrollment of the student. With this research it was found that both students and teachers to the Social Networking Sites ( SNS ) is presented as an educational technology tool, serving to: search the contents; perform school tasks ; communicate, send and receive materials via e- mail service. Furthermore, the SRS advance and extend possibilities of communication and interaction. This text is divided into an introductory part on the student's contemporaneity, a debate on the use of SRS in teaching and learning, an account of the main findings of the research and final considerations.

Keywords: Teaching and Learning; Social Networking Sites; education; digital natives
\end{abstract}

1 Dados do grupo disponíveis em http://plsql1.cnpq.br/buscaoperacional/detalhegrupo.jsp?grupo=0045708S2ENU4Y

V. $12 \mathrm{~N}^{\mathrm{o}} 1$, julho, 2014 


\section{Introdução}

As mídias sociais estão presentes na vida cotidiana dos estudantes, merecendo destaque a expressiva utilização dos Sites de Redes Sociais (SRS) como uma forma de sociabilidade. A pesquisa realizada buscou mapear os alunos da Universidade Federal de Pelotas (UFPel), nativos digitais, nascido após 1990, bem como o uso das mídias digitais com foco nos SRS. Este texto, baseado nos resultados da pesquisa procura problematizar o uso deste dispositivo como apoio ao ensino e aprendizagem e construção de conhecimento no ensino superior. Será apresentada uma análise das respostas encontradas a partir do questionário online, disponibilizado para os alunos da UFPel, durante o processo de realização das rematrículas do segundo semestre de 2013.

Neste sentido, esta pesquisa foca temas ligados às transformações contemporâneas em relação aos novos modos de aprendizagens, produção de conhecimentos e sociabilidades produzidas no ambiente online e, sobretudo os SRS, apontam indícios de que possam atuar como propulsores e catalisadores destas transformações por meio dos artefatos midiáticos digitais utilizados pelos acadêmicos da UFPel.

O conhecimento da forma de acesso às mídias digitais pelos alunos, indica aos professores da UFPel e aos professores em geral, possibilidades do uso de mídias digitais para o ensino e aprendizagem. Vive-se em uma sociedade da informação, é preciso repensar as práticas educativas (SIBÍLIA, 2012).

Da intensificação dos processos interativos (online) entre as pessoas, emergem características de aprendizagens e de sociabilidades não existentes antes da internet. Constata-se hoje que as tecnologias digitais contemporâneas, por sua rápida evolução, e por serem mais acessíveis e atraentes, potencializam os números de conexões, contatos e interações online. A quantidade de informações se avoluma em tempos cada vez menores.

Conforme (SPEROTTO, 2011), tais mudanças alteram os modos de existência e outras constituições subjetivas estão sendo engendradas no contemporâneo. Bem como, a produção do conhecimento, as interações online e as aprendizagens, constituem uma subjetividade que é individual e coletiva (GUATTARI,1992). Ousamos afirmar que, as pessoas estão sendo constituídas em meio a esses dispositivos tecnológicos que se apresentam diferentes dos que existiam antes do advento da proliferação das mídias digitais, o que implica no surgimento de outras possibilidades de aprendizagens. Tratase de aprendizagens interativas. A aprendizagem interativa e coletiva é uma realidade contemporânea, onde a internet produz, enfim, novas subjetividades e aprendizagens (GUATTARI,1999). A internet é um instrumento de desenvolvimento social que possibilita a partilha da memória, da percepção, da imaginação e das sensações, resultando numa aprendizagem coletiva, advindas de explorações entre grupos humanos, destaca (LEVY, 1997).

\section{Procedimentos Metodológicos}

Como pressuposto metodológico utiliza-se a etnografia virtual como método de captura e de coleta destes dados, cuja ênfase está numa proposta de investigação na internet que, enriquece as vertentes da perspectiva de inovação e melhoramento social dentro do espectro do enfoque qualitativo de metodologia e prática social (FRAGOSO; RECUERO; AMARAL, 2011; KOZINETS, 2010). Resulta do trabalho de campo que 
estuda as culturas e comunidades online emergentes, mediadas por computador, ou comunicações baseadas na internet, requerem métodos de "pesquisa online" adaptados e pertinentes a esta realidade. Independente do termo utilizado. Um fato interessante a considerar é que, o incremento de pesquisas de cunho antropológico no ciberespaço é um sinal de que a internet não é mais apenas considerada um artefato cultural passando a ser considerada também como um contexto cultural.

A etnografia virtual pode ser utilizada de três maneiras: a) como metodologia para estudar comunidades virtuais puras; b) como metodologia para estudar comunidades virtuais derivadas; c) como ferramenta exploratória para estudar diversos assuntos (KOZINETS, 1997). No caso da pesquisa, que é foco deste artigo, ela foi realizada a partir da disponibilidade de um instrumento online, que possibilitou a cada estudante, no momento de realizar sua matricula no sistema da UFPel, respondesse a este questionário, que contemplou perguntas sobre mídias digitais e o uso para a construção do conhecimento. Sobre os sujeitos da pesquisa: das 11.006 solicitações de matrícula dos alunos das diferentes graduações da UFPEL (Bacharelado e Licenciaturas), obtivemos 5.178 formulários respondidos, desses 2.643 são de sujeitos nascidos a partir de 1990, ou seja, nativos digitais (PRENSKY, 2013).

\section{Principais Contribuições deste Estudo}

Os resultados obtidos na investigação poderão servir como subsídios teóricos para serem utilizados como ferramentas de problematização, tanto para estudantes quanto para professores de diferentes níveis de ensino, uma vez que apresentaremos possibilidades de reconhecer os modos como os estudantes pesquisados transitam "entre e com" as mídias socais.

Da mesma forma, os desdobramentos da pesquisa podem contribuir para a reflexão crítica sobre novas práticas e metodologias que contemplem o uso das mídias sociais como ferramentas de ensino-aprendizagem, indicando possibilidades de ações dos professores junto aos estudantes. "Essa nova geração de nativos digitais possui uma identidade virtual, pois passam a maior parte do tempo conectados através das redes sociais, blogs, jogos online, em meio às inovações tecnológicas" (SANTOS, 2011 p.15844).

Uma das análises realizadas a partir dos dados da pesquisa foi em relação aos dispositivos utilizados para acessar a rede internet e, especificamente, os SRS. Os dados dos alunos nascidos a partir de 1990 indicam que $84,45 \%$ acessam a internet e os SRS pelo computador portátil (notebook). Dos entrevistados $97,5 \%$ afirmam utilizarem SRS e mais de 80,9\% conectam-se por Smartphone ou Tablet, outro dado importante é que destes, $36 \%$ conectam-se a internet gratuita enquanto $27,4 \%$ pagam para manter-se conectado com a mídia de cultura mobile.

Outra questão realizada foi em relação à quantidade de horas por dia, em média, que o aluno permanece na rede.

Em todos os grupos a maioria dos respondentes indicou de $1 \mathrm{~h}$ a $3 \mathrm{~h}$ de interação, ficando em segundo lugar de $3 \mathrm{~h}$ a $6 \mathrm{~h}$. 


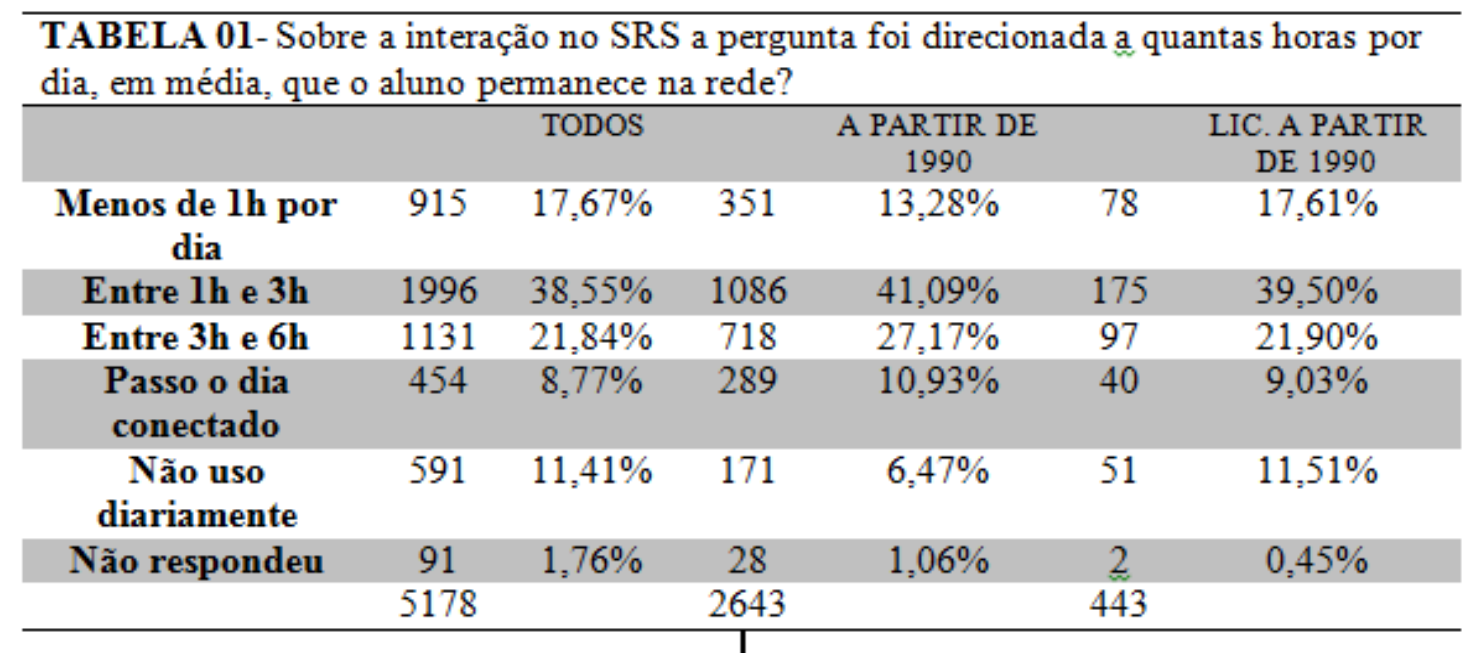

Fonte: Tabela elaborada com base nos dados coletados na pesquisa.

Quando perguntados sobre o motivo pelo qual não utilizam o SRS com mais frequência, a maioria dos respondentes $(53,94 \%)$ afirmou que isso ocorre em função da falta de tempo. O cuidado em não expor sua vida é a preocupação de $26,52 \%$ dos entrevistados. A falta de privacidade da rede social aparece como terceiro motivo pelo qual os alunos não utilizam com mais frequência o SRS, opção de 8,05\%. 6,35\% não especificaram seus motivos, seguidos de $3,90 \%$ dos respondentes que tem medo dos riscos que a rede pode oferecer. Entre os nascidos a partir de 1990 as respostas não foram significativamente diferentes, tendo, na maior parte das opções uma variação de menos de $1 \%$, tanto para mais como para menos. Da mesma forma, não houve uma diferença significativa entre os nativos digitais matriculados em cursos de licenciatura.

\begin{tabular}{|c|c|c|c|c|c|c|}
\hline \multicolumn{7}{|c|}{ TABELA 02 - Porque não utiliza o SRS } \\
\hline & & TODOS & & $\begin{array}{l}\text { A } \\
\text { PARTIR } \\
\text { DE } 1990\end{array}$ & & $\begin{array}{l}\text { LIC. A } \\
\text { PARTIR } \\
\text { DE } 1990\end{array}$ \\
\hline $\begin{array}{l}\text { Não gosto } \\
\text { de expor } \\
\text { minha vida. }\end{array}$ & 1373 & 26,52 & 713 & 26,98 & 109 & 24,60 \\
\hline $\begin{array}{l}\text { Tenho medo } \\
\text { dos riscos } \\
\text { que a rede } \\
\text { pode } \\
\text { oferecer. }\end{array}$ & 202 & 3,90 & 85 & 3,22 & 18 & 4,06 \\
\hline $\begin{array}{l}\text { Falta de } \\
\text { privacidade. }\end{array}$ & 417 & 8,05 & 226 & 8,55 & 24 & 5,42 \\
\hline $\begin{array}{l}\text { Falta de } \\
\text { tempo. }\end{array}$ & 2793 & 53,94 & 1398 & 52,89 & 257 & 58,01 \\
\hline $\begin{array}{l}\text { Outros } \\
\text { motivos }\end{array}$ & 329 & 6,35 & 224 & 8,48 & 47 & 10,61 \\
\hline
\end{tabular}

Fonte: Tabela elaborada com base nos dados coletados na pesquisa. 
Ao questionar com que finalidade os alunos utilizam o SRS, a pesquisa buscou entender o que aproxima estes usuários da rede, e sabendo do seu interesse e sua permanência, estes dão pistas de como aproximar o ensino e a aprendizagem da rotina virtual do aluno.

\begin{tabular}{lcccccc}
\hline \multicolumn{2}{l}{ TABELA 03 - Com que finalidade utilizam a rede } \\
\hline & TODOS & $\begin{array}{c}\text { A PARTIR } \\
\text { DE 1990 }\end{array}$ & $\begin{array}{c}\text { LIC. A PARTIR } \\
\text { DE 1990 }\end{array}$ \\
$\begin{array}{l}\text { Para encontrar } \\
\text { amigos }\end{array}$ & 3842 & $74,20 \%$ & 2115 & $80,02 \%$ & 328 & $74,04 \%$ \\
$\begin{array}{l}\text { Para } \\
\text { entretenimento. }\end{array}$ & 2598 & $50,17 \%$ & 1503 & $56,87 \%$ & 248 & $55,98 \%$ \\
$\begin{array}{l}\text { Para visualizar } \\
\text { notícias. }\end{array}$ & 2383 & $46,02 \%$ & 1285 & $48,62 \%$ & 214 & $48,31 \%$ \\
$\begin{array}{l}\text { Para estudar. } \\
\text { Outros motivos }\end{array}$ & 1488 & $28,74 \%$ & 802 & $30,34 \%$ & 155 & $34,99 \%$ \\
\hline
\end{tabular}

Fonte: Tabela elaborada com base nos dados coletados na pesquisa.

Entende-se que o acesso é tão importante quanto à permanência no SRS, tendo em vista as ações dos alunos na rede, e o tempo que é consumido nesta tarefa. Muitas questões estão envolvidas, até pensar que pode ser uma fuga das tarefas ditas chatas do estudo, assim este dado revela com o aluno gerencia o seu tempo.

Observa-se que 34,99\% dizem que utilizam o SRS para estudar, e 48,31\% para manter-se atualizados com notícias, enquanto $56,87 \%$ para entretenimento, assim é possível afirmar que, o maior interesse na rede está diretamente relacionado ao lazer².

Dos alunos que dizem interagir no SRS, questionou-se o que ocorre que o leva a compartilhar algo no Facebook?

Conforme a tabela 04 observa-se que a identificação com o assunto é o ponto que mais impulsiona o curtir no SRS.

Frente às possibilidades de comunicação com os alunos pelos SRS, apresentamse os grupos que podem ser criados pelo professor de forma aberta ou fechada (só a turma visualiza) e por esta ferramenta alargar o tempo de aula para os debates e compartilhar materiais diversos relacionados à disciplina ou não.

Pensando em entender como o aluno utiliza esta ferramenta questionou-se: Quais tipos de grupos você participa (assuntos)?

2 Lazer é uma forma de se libertar das ocupações do dia-a-dia, seja, do trabalho assalariado, e das obrigações diárias, é se satisfazer com algo que esteja relacionados em dois aspectos, tempo livre (não trabalho) e atitude (prazer),ou seja, atividades que esteja fora do tempo das ocupações diárias em geral e ao mesmo tempo que Ihe proporcione prazer e sensação de liberdade. (MARCELINO, 2000) 


\begin{tabular}{|c|c|c|c|c|c|c|}
\hline \multicolumn{7}{|c|}{ TABELA 05 -Participação em grupos no SRS } \\
\hline & & \multicolumn{2}{|c|}{ TODOS } & \multicolumn{2}{|c|}{$\begin{array}{l}\text { A } \\
\text { PARTIR } \\
\text { DE } 1990\end{array}$} & $\begin{array}{l}\text { LIC. A } \\
\text { PARTIR } \\
\text { DE } 1990\end{array}$ \\
\hline Esporte. & 1400 & 27,04 & 774 & 29,28 & 97 & 21,90 \\
\hline Cultura. & 2364 & 45,65 & 1248 & 47,22 & 245 & 55,30 \\
\hline Tecnologia. & 1286 & 24,84 & 742 & 28,07 & 86 & 19,41 \\
\hline Acadêmico. & 3969 & 76,65 & 2161 & 81,76 & 349 & 78,78 \\
\hline $\begin{array}{l}\text { Não } \\
\text { participo } \\
\text { de grupos } \\
\text { no } \\
\text { Facebook. }\end{array}$ & 380 & 7,34 & 117 & 4,43 & 27 & 6,09 \\
\hline Outro: & 306 & 5,91 & 144 & 5,45 & 33 & 7,45 \\
\hline
\end{tabular}

Fonte: Tabela elaborada com base nos dados coletados na pesquisa.

De acordo com a tabela 05 , os números de alunos que se interessam pelos grupos acadêmicos são de $76,65 \%$. Desta forma, o estudo indica a possibilidade de uso do SRS pelos professores com finalidade de ensino e aprendizagem. Porém é importante destacar que, não basta transferir a aula presencial para online, certamente não irá funcionar, pois existe a necessidade de experimentar os recursos que irão variar de turma para turma, de aluno para aluno.

A tabela 06 apenas reafirma a possibilidade do uso de forma acadêmica, pois mais de $80 \%$ dos alunos concordam que os SRS podem ser utilizados para aprendizado.

\begin{tabular}{lcccccc}
\hline \multicolumn{1}{c}{ TABELA 06 - O SRS pode ser utilizado para aprender conteúdos do seu curso } \\
\hline \multicolumn{3}{c}{ TODOS } & A PARTIR DE 1990 & LIC. A PARTIR DE 1990 \\
Sim & 4354 & 84,09 & 2315 & 87,59 & 392 & 88,49 \\
Não & 824 & 15,91 & 328 & 12,41 & 51 & 11,51 \\
\hline
\end{tabular}

Fonte: Tabela elaborada com base nos dados coletados na pesquisa.

Procurou-se obter a percepção dos discentes em relação à utilização do SRS como uma ferramenta de ensino-aprendizagem. Pelo elevado percentual de respostas positivas (respectivamente $84,09 \%, 87,59 \%$ e $88,49 \%$ ), na percepção dos alunos, é possível utilizar o SRS para proporcionar aprendizado. É interessante observar que, a faixa de alunos nascidos a partir de 1990 dos cursos de licenciaturas são os que mais acreditam nesta possibilidade, com um percentual acima de $88 \%$.

O SRS é um ambiente adequado para ensino-aprendizagem comparado ao Ambiente Virtual de Aprendizagem AVA Moodle, que é utilizado nas universidades públicas, incluindo a UFPel. Diferentemente do AVA, o SRS é um ambiente em que os alunos o utilizam com naturalidade, não vinculado a um ambiente de estudo, entretanto podendo ser explorado pelos professores para a produção de conhecimento. 
Produzir de forma descentralizada e de maneira não-formatada ou preconcebida. Produzir e ocupar os espaços, todos os espaços, através das redes. Nesse contexto, a apropriação da cultura digital passa a ser fundamental, uma vez que ela já indica intrinsecamente um processo crescente de reorganização das relações sociais mediadas pelas tecnologias digitais. (PRETO, 2000 p.78)

É interessante observar que em uma pesquisa desenvolvida pelo Grupo de pesquisa: Prática Pedagógica no Ensino e Aprendizagem com Tecnologias Educacionais (PRAPETEC) da Pontifícia Universidade Católica do Paraná PUCPR Ferreira et all (2012 p.10) "Você utilizaria o Facebook como um ambiente virtual de aprendizagem?"

SIM.

Segundo os relatos, os 25 alunos participantes da pesquisa responderam que

\begin{tabular}{lcccccc}
\hline $\begin{array}{l}\text { TABELA 07-Se con cord as com o uso do SRS para o ensino e apren dizagem } \\
\text { respond a de que maneira? }\end{array}$ & & TODOS & $\begin{array}{c}\text { A PARTIR } \\
\text { DE } 1990\end{array}$ & $\begin{array}{c}\text { LIC. A } \\
\text { PARTIR DE } \\
1990\end{array}$ \\
\hline & 895 & 17,28 & 537 & 20,32 & 80 & 18,06 \\
$\begin{array}{l}\text { Tirando dúvidas de } \\
\text { conteúd os }\end{array}$ & 1419 & 27,40 & 765 & 28,94 & 123 & 27,77 \\
$\begin{array}{l}\text { Aumentando a interação } \\
\text { do grupo }\end{array}$ & 562 & 10,85 & 280 & 10,59 & 52 & 11,74 \\
$\begin{array}{l}\text { Como ferramenta de } \\
\text { pesquisa e fonte de } \\
\text { conteúdo complementar }\end{array}$ & 1498 & 28,93 & 743 & 28,11 & 140 & 31,60 \\
$\begin{array}{l}\text { Tod as as alternativas } \\
\text { acima }\end{array}$ & 804 & 15,53 & 318 & 12,03 & 48 & 10,84 \\
$\begin{array}{l}\text { Não responderam } \\
\text { Outro: }\end{array}$ & 77 & 1,49 & 39 & 1,48 & 7 & 1,58 \\
\hline
\end{tabular}

Fonte: Tabela elaborada com base nos dados coletados na pesquisa.

Em relação às formas de aprendizagem utilizando o SRS, houve certa prevalência (entre $28 \%$ e $31 \%$ ) dos respondentes em afirmar que "todas as alternativas" são possibilidades de aprendizagem. Porém, dentre as alternativas, podemos dizer que, a maneira "Aumentando a interação do grupo" foi destacada entre aqueles que escolheram uma das alternativas.

Demonstrar a percepção da importância das conexões e troca de informações entre os integrantes de uma comunidade de aprendizagem, prevalecendo sobre "tirar dúvidas de conteúdos" e "ferramenta de pesquisa", também vai ao encontro do próprio objetivo do SRS. 


\section{Considerações Finais}

Para os alunos do contemporâneo, nativos digitais, usuários dos SRS, encontrouse uma aceitação da ferramenta para o ensino e aprendizagem, porém ela aparece relacionada com a interação entre o grupo com força, se comparado ao item sanar dúvidas pelo professor. Trata-se de uma aprendizagem em rede, na qual o grupo de alunos trama e tece o conhecimento.

Constatou-se que, tanto para os estudantes como para os professores, o SRS apresenta-se como uma ferramenta tecnológica pedagógica, servindo para: pesquisar conteúdos; realizar tarefas escolares; comunicar-se, enviar e receber materiais através do serviço de e-mail. Percebe-se ainda que, não apenas como pesquisador, mas na prática diária como usuário destas tecnologias, que avançam e alargam as possibilidades de comunicação e interação.

\section{Referências Bibliográficas}

DELEUZE, Gilles; GUATTARI, Félix. Mil platôs: capitalismo e esquizofrenia. Vol. 1. Rio de Janeiro. Ed. 34, 1995.

FRAGOSO, Suely; RECUERO, Raquel; AMARAL, Adriana. Métodos de Pesquisa para internet. Porto Alegre: Sulina, 2011, 239p.

FERREIRA, J. L.; CORREAA, B. R. P. G.; TORRES, P. L. O uso pedagógico da rede social Facebook. In: TORRES, P. L.; WAGNER, P. R. Redes Sociais e Educação: desafios contemporâneos. Porto Alegre: EDIPUCRS, 2012. Disponível em: <http://www.ead.pucrs.br/ebook-ricesu2012/>

GUATTARI, Félix. Caosmose: um novo paradigma estético. Tradução de Ana Lúcia de Oliveira e Lúcia Cláudia Leão. Rio de Janeiro: Ed. 34, 1992.

GUATTARI, Felix. Da Produção de Subjetividade. In: PARENTE, A. (org.) Imagem Máquina: A Era das Tecnologias do Virtual. Rio de Janeiro: 34, 1999.

KOZINETS, Robert V. Netnography- Doing Ethnographic Research on-line. Londres: SAGE, 2010.

KOZINETS, Robert. Netnography 2.0. In: R. W. BELK, Handbook of Qualitative Research Methods in Marketing. Edward Elgar Publishing, 1997.

LÉVY, Pierre. Cibercultura. São Paulo: Ed. 34, 1997.

MARCELLINO, Nelson. C. . O lazer na atualidade brasileira: perspectivas

na formaçãolatuação profissional.. Licere, CELAR-UFMG, v. 3, p. 125-133, 2000.

MARGARITES, Ana.P. F, Subjetividade e Redes Sociais na internet: As relações entre estudantes e professores no contemporâneo. 2011. 116f. Dissertação (Mestrado em Educação) Faculdade de Educação. Universidade Federal de Pelotas. Pelotas, 2011. PRETTO, Nelson L., and SILVEIRA, SA., orgs. Além das redes de colaboração: internet, diversidade cultural e tecnologias do poder. [online]. Salvador: EDUFBA, 2008. 232 p. ISBN 978-85-232-0524-9. Available from SciELO Books $<$ http://books.scielo.org>. Acesso em 07 de maio de 2014.

PRENSKY, Marc. Digital Natives, Digital Immigrants. Disponível em: http://goo.gl/4oYb> Acesso em: 5 Abril de 2013.

ROLNIK, Suely. Cartografia Sentimental: Transformações Contemporâneas do Desejo.Porto Alegre:Sulina, 2006. 
SANTOS, M. SCARABOTTO, S. MATOS, E. Imigrantes e Nativos Digitais: Um Dilema OU Desafio na Educação? X CONGRESSO DE EDUCAÇÃO EDUCARE I SEMINÁRIO INTERNACIONAL DE REPRESENTAÇÕES SOCIAIS, SUBJETIVIDADE E EDUCAÇÃO SIRSSE PUC-PR 2011.

SPEROTTO, Rosária. I. . Comunicação, Cultura e Tecnologias: Possibilidades de Pesquisas na Educação. In: Paula Corrêa Henning; Paula Regina Costa Ribeiro; Elisabeth Brandão Schmidt. (Org.). Perspectivas de Investigação no campo da Educação Ambiental \& Educação em Ciências. $1^{\text {a }}$ ed. Rio Grande: Ed. da FURG, 2011, v. 0, p. 01118. 